Published in final edited form as:

Am J Obstet Gynecol. 2018 April ; 218(4): 425.e1-425.e18. doi:10.1016/j.ajog.2017.12.218.

\title{
Nationwide Trends in the Utilization of and Payments for Hysterectomy in the United States Among Commercially Insured Women
}

\author{
Daniel M. MORGAN, MD¹, Mr. Neil S. KAMDAR, MS ${ }^{1}$, Carolyn W. SWENSON, MD ${ }^{1}$, Ms. Emily \\ K. KOBERNIK, MPH ${ }^{1}$, Anne G. SAMMARCO, MD ${ }^{1}$, and Brahmajee NALLAMOTHU, MD, MPH ${ }^{2}$ \\ ${ }^{1}$ University of Michigan Department of Obstetrics \& Gynecology, Ann Arbor, MI \\ 2University of Michigan Institute for Healthcare Policy \& Innovation, Ann Arbor, MI
}

\begin{abstract}
Background-Laparotomy followed by inpatient hospitalization has traditionally been the most common surgical care for hysterectomy. The financial implications of the increased use of laparoscopy and outpatient hysterectomy are unknown.

Objectives-To quantify the increasing use of laparoscopy and outpatient hysterectomy and to describe the financial implications among women with commercially based insurance in the United States.
\end{abstract}

Study Design-Hysterectomies between 2010 and 2013 were identified in the Health claims for more than 25 million women. Surgical approach was categorized with Care Cost Institute, a national dataset with inpatient and outpatient private insurance procedure codes as abdominal, laparoscopic, laparoscopic assisted vaginal, or vaginal. Payments were adjusted to 2013 U.S. dollars to account for change due to inflation.

Results-Between 2010 and 2013, there were 386,226 women who underwent hysterectomy. The rate of utilization decreased $12.4 \%$, from 39.9 to 35.0 hysterectomies per 10,000 women. The largest absolute decreases were observed among women less than 55 years and among those with uterine fibroids, abnormal uterine bleeding, and endometriosis. The proportion of laparoscopic hysterectomies increased from $26.1 \%$ to $43.4 \%$, with concomitant decreases in abdominal (38.6\% to $28.3 \%$ ), laparoscopic assisted vaginal (20.2 to $16.7 \%$ ), and vaginal (15.1\% to $11.5 \%)$ hysterectomies. There was also a shift from inpatient to outpatient surgery. In 2010, the inpatient and outpatient rates of hysterectomy were 26.6 and 13.3 per 10,000 women, respectively. By 2013 , the rates were 15.4 and 19.6 per 10,000 women. In each year of analysis, the average reimbursement for outpatient procedures was $44-46 \%$ less than for similar inpatient procedures.

Corresponding Author: Daniel M. Morgan, MD University of Michigan Department of Obstetrics \& Gynecology, 1500 East Medical Center Dr. Ann Arbor, Michigan, 48109 USA morgand@umich.edu Phone: 734-647-5866.

Publisher's Disclaimer: This is a PDF file of an unedited manuscript that has been accepted for publication. As a service to our customers we are providing this early version of the manuscript. The manuscript will undergo copyediting, typesetting, and review of the resulting proof before it is published in its final citable form. Please note that during the production process errors may be discovered which could affect the content, and all legal disclaimers that apply to the journal pertain.

Previous Presentation: This work was presented at the Society for Gynecologic Surgeons on March 27, 2017 in San Antonio, Texas. Conflicts of Interest: The authors report no conflict of interest. 
Offsetting the lower utilization of hysterectomy and lower reimbursement for outpatient surgery were increases in average inpatient and outpatient hysterectomy reimbursement of $19.4 \%$ and $19.8 \%$, respectively. Total payments for hysterectomy decreased $6.3 \%$, from $\$ 823.4$ million to $\$ 771.3$ million.

Conclusion-Between 2010 and 2013, laparoscopy emerged as the most common surgical approach for hysterectomy, and outpatient hysterectomy became more based insurance. While common than inpatient among women with commercially average reimbursement per case increased, overall payments for hysterectomy are decreasing due to decreased utilization and dramatic differences in how hysterectomy is performed.

\section{Keywords}

hysterectomy; laparoscopy; reimbursement; trends

\section{Introduction}

Utilization of hysterectomy, the second most common procedure performed among women in the United States, is rapidly evolving. ${ }^{1}$ National claims data in one study revealed that the median number of inpatient hysterectomies per hospital decreased by $40 \%$ over 10 years. $^{2}$ However, it is possible that the dissemination of minimally invasive hysterectomy techniques, especially laparoscopy, has led to protocols promoting outpatient hysterectomy.

3-5 Because ambulatory data are often not included in large national claims datasets, it is unclear if the declines in inpatient hysterectomy are due to an overall decrease in hysterectomy utilization or a shift toward outpatient care.

The financial impact of these changes in practice is unclear. The widespread adoption of laparoscopy - in particular, robotic assisted technology — could have profound financial repercussions. The American College of Obstetricians and Gynecologists (ACOG) estimated that robotic hysterectomy could add $\$ 900$ million to $\$ 1.9$ billion to annual healthcare spending. ${ }^{6}$ The financial implications of these changes in practice have not been evaluated among commercially insured women.

The Health Care Cost Institute- a non-partisan, non-profit organization-contains inpatient and outpatient claims data from three large commercial insurers. It is well-suited for an analysis of care received by U.S. women in their fourth, fifth, and sixth decades-a time of life when most women are covered by private insurance and when hysterectomy is most commonly performed. We sought to quantify the emergence of laparoscopy as the predominant surgical approach for hysterectomy, to describe the shift from inpatient to outpatient surgery, and to analyze the financial implications of these changes. This analysis seeks to provide transparency to patients, hospitals, and insurers regarding the diffusion of technology and its impact on utilization and payment for this common surgery.

\section{Materials and Methods}

We used data from the Health Care Cost Institute, a repository for inpatient and outpatient claims for more than 25 million women whose health insurance coverage was provided by 
Aetna, Humana, and UnitedHealthcare (http://www.healthcostinstitute.org). The study was deemed "not regulated" by the University of Michigan Institutional Review Board (HUM00114432).

Hysterectomies between January 1, 2010 and December 31, 2013 were identified using procedure codes of the International Classification of Diseases Version 9 (ICD9) and Current Procedural Terminology (CPT4) systems. The surgical approach for hysterectomy was determined primarily with ICD9 procedure codes; when ICD9 codes were not available, a CPT4 code was used instead. When cases had more than one surgical approach indicated by these codes, the surgical approach was categorized with the less invasive approach. We chose to categorize cases in this manner to be consistent with an intent to treat analysis. Clinically, it is most common that a surgery would transition from a minimally invasive to an open approach in the event of unexpected pathology or complications. A case involving the surgical judgment that conversion is required will involve greater resource use (e.g. additional instruments, more surgical time, and inpatient admission) and will result higher payment, even in the absence of complications. Using the intent to treat principle, this resource use is attributed to the less invasive, less morbid outpatient approach rather than the already comparatively more morbid, inpatient abdominal approach. Thus, if vaginal and abdominal procedure codes were present, the case was analyzed with the vaginal surgical approach group. If both laparoscopic and abdominal approaches were present, the case was analyzed with the laparoscopic surgical approach group. Procedures with a robotic assisted ICD9 procedure code $(17.42,17.44)$ were considered laparoscopic hysterectomies.

Surgical indications for hysterectomy were identified using ICD9 diagnostic codes. Three diagnostic codes were available to allow for identification of hysterectomies for multiple indications. In alphabetical order, the indications were: abnormal uterine bleeding (AUB); cancers of the cervix, uterus, ovary, unspecified female organ; cervical dysplasia; endometrial hyperplasia; endometriosis, non-inflammatory disorders \& pelvic pain; family or personal history of malignancy; leiomyomata; obstetrical complications; pelvic organ prolapse; pelvic inflammatory disease; premalignant lesions or tumors of uncertain behavior. The ICD9 codes used in each of these groups are provided in Supplementary Table 1.

Utilization - the number of women per 10,000 who underwent hysterectomy-was analyzed with adjustments for state-level variation in the proportion of women in the Health Care Cost Institute and differences in age across states. A patient's residence was determined by their location at the beginning of the calendar year. The number of hysterectomies among women enrolled in the Health Care Cost Institute was tallied for each state in each calendar year. To estimate the number of hysterectomies in any state, we took into account how many women had insurance with one of the three carriers in the Health Care Cost Institute and the number of women in that respective state with commercially based insurance according to the United States census. Thus, the state count of hysterectomies was divided by the ratio of the number of patients covered by one of the three insurance carriers over the number with commercially based insurance. Indirect age adjustment, as described by the Dartmouth Atlas of Healthcare, was used to account for the differing proportions of women in age bands 18-24, 25-34, 35-44, 45-54, 55-64, 65-74, and > 75 years. ${ }^{7}$ Age-adjusted rates for inpatient, 
outpatient, and overall (both inpatient and outpatient cases) utilization were then calculated at the state level.

We also analyzed changes between the bookend years of 2010 and 2013. The overall percent difference in rates of hysterectomy for each state between 2010 and 2013 was calculated and ranked from largest to smallest relative decrease. The state rates for 2010 and 2013 were combined and the decile limits for inpatient, outpatient, and overall utilization were each identified. State utilization rates were mapped to show the relative changes across the states. Box plots showing the median and interquartile range were developed to illustrate the trends in state-level utilization rates of hysterectomy between 2010 and 2013.

Payments were analyzed after adjustment to 2013 U.S. dollars using the medical consumer price index. Facility payments and patient cost share payments (i.e., out-of-pocket expenses) for the hysterectomy, provided by the Health Care Cost Institute, were used in the analysis. The facility payment is the amount reimbursed by the insurance company to the hospital, and the patient cost share is the sum of coinsurance, copayments, and deductibles made by the patient. Total payment is the sum of the facility payment and patient cost share.

\section{Results}

We identified 386,226 women who underwent hysterectomy during 102,747,857 womanyears of observation in the Health Care Cost Institute data. Uterine fibroids, abnormal uterine bleeding, and endometriosis were the three most common indications for surgery. $78.3 \%(\mathrm{n}=302,547)$ of the hysterectomies were performed on women $<55$ years of age. The utilization of hysterectomies decreased $12.3 \%$ overall. In the four calendar years, the rate declined from 39.9, to 39.0, to 36.5, to 35.0 hysterectomies per 10,000 women (Table 1). The annualized average decrease in hysterectomy utilization was $4.3 \%$.

Patient demographics and diagnoses between 2010 and 2013 were analyzed (Table 1). Among women ages $<35,36-44$, and $45-54$ years, there were decreases in utilization of $22.2 \%, 16.9 \%$, and $15.2 \%$, respectively. In contrast, the utilization of hysterectomy among women ages 55-64 decreased only 0.3\%, and among women ages 65-74 and $>74$, the rate increased $17.1 \%$ and $1.3 \%$, respectively. The use of ICD9 codes for uterine fibroids, abnormal uterine bleeding, and endometriosis decreased $11.4 \%, 17.7 \%$, and $9.4 \%$, respectively. The use of pelvic organ prolapse and pelvic inflammatory disease-the fourth and fifth most common diagnoses-decreased $17 \%$ and $20 \%$. The proportion of hysterectomies for uterine cancer increased $4 \%$, while the proportion performed for ovarian cancer, cervical cancer, poorly specified cancers, pre- malignant conditions (cervical dysplasia, endometrial hyperplasia), and a family history of cancer were relatively stable.

Choice of surgical approach, payments made by insurance companies and patients, and facility reimbursement for hysterectomy changed substantially between 2010 and 2013 (Table 1). The proportion of hysterectomies performed laparoscopically increased from $26.1 \%$ to $43.4 \%$. In 2012, laparoscopy emerged as the most common surgical approach for hysterectomy. There were concomitant decreases in the proportion of non-laparoscopic hysterectomies, which include abdominal, vaginal, and laparoscopic assisted vaginal cases. 
There was a $34.1 \%$ decline in the proportion of hysterectomies performed inpatient and a small decrease in average length of stay. The payment for and utilization of hysterectomy in the inpatient and outpatient setting in each of the four years is illustrated in Figure 1.

Between 2010 and 2013, reimbursement for outpatient hysterectomy was $44 \%$ to $46 \%$ lower than that for inpatient (e.g., in 2010: $(\$ 5,100-\$ 9,451) / \$ 9,451 * 100 \%)$. The average increase in payment per hysterectomy was $19.4 \%$ (e.g., $(\$ 11,284-\$ 9,451) / \$ 9,451 * 100 \%$ ) for inpatient and $19.8 \%$ (e.g., $(\$ 6,112-\$ 5,100) / \$ 5,100 * 100 \%$ ) for outpatient. In 2010, inpatient hysterectomy was twice as common as outpatient. With each year, the gap narrowed, and in 2013, outpatient hysterectomy became more common than inpatient.

Trends in the average payment for inpatient and outpatient hysterectomy by each surgical approach are provided in Figure 4. The per case payments for hysterectomy, adjusted for inflation, increased for all surgical approaches and for both inpatient and outpatient cases between 2010 and 2013. In comparison to a vaginal approach, there is an additional expense associated with laparoscopy (both total laparoscopic and laparoscopic assisted vaginal hysterectomy) for inpatient and outpatient hysterectomy. Payments for abdominal hysterectomies, preformed predominantly inpatient, were similar to those for hysterectomy performed with laparoscopy. The proportion of outpatient abdominal hysterectomies is $<1 \%$ of hysterectomies in all years analyzed.

Aggregated at the state level, there is evidence of decreasing geographic variation in hysterectomy rates. Three pairs of United States maps illustrate the changes between 2010 and 2013. (Note: calculations for age-adjusted utilization rates for each state are provided in Supplementary Tables 2 and 3). The green shade for inpatient hysterectomy lightens, indicating decreased utilization, while the blue shade for outpatient hysterectomy darkens, reflecting increased utilization. For overall rates, there is a lightening of the purple shade, reflecting lower rates of hysterectomy. The median decrease in hysterectomy utilization among states was $-13.3 \%$ and the range was $-25.8 \%$ to $-6.7 \%$. The boxplot (see Figure 5) illustrates a $12.3 \%$ drop in the median state-level hysterectomy rate (consistent with that reported in Table 1) and a 48.5\% decrease in the interquartile variance between 2010 and 2013 (0.0723 vs. $0.0372, \mathrm{p}=.01$ ).

The total annual payments for hysterectomy were analyzed over this time. Several factors are responsible for the $6.9 \%$ decrease in payments ( $\$ 823$ million to $\$ 771$ million) between 2010 and 2013 (Table 1). Lower total annual payments were related primarily to a $12.3 \%$ decrease in utilization and a shift from inpatient to outpatient surgery, for which average case reimbursement is approximately $45 \%$ less. The effects of these factors were partially offset by increases in average inpatient (19.4\%) and outpatient (19.8\%) reimbursement. The effect of laparoscopy and outpatient surgery usage rates on total annual payments between 2010 and 2013 is illustrated in Figure 3. For outpatient hysterectomy, there were increases in payments for laparoscopic and non-laparoscopic approaches of \$113 million (131\%) and \$26 million (28\%), respectively. In contrast, for inpatient hysterectomy, there were decreases in payments for laparoscopic and non-laparoscopic approaches of \$16 million (14\%) and $\$ 175$ million (32\%). The average total payment for all hysterectomies (inpatient and outpatient) rose $4.8 \%$ (\$387), with the patient cost share increasing $11.9 \%$ (\$84 per case) and the insurance payment increasing $4.2 \%$ ( $\$ 303$ per case). 


\section{Comment}

In this analysis of more than 25 million commercially insured women, laparoscopy became the most common surgical approach for hysterectomy between 2010 and 2013. The proportion of hysterectomies performed laparoscopically increased $66.3 \%$. The rapid growth in use of laparoscopy builds upon years of steady, albeit slower increases in utilization. ${ }^{2,8,9}$ The proportion of hysterectomies performed outpatient increased $67.8 \%$, and outpatient hysterectomy became more common than inpatient in 2013. With the majority of hysterectomies now performed outpatient, epidemiologic analyses of hysterectomy in the future will need to consider inpatient and outpatient procedures.

The increased use of laparoscopy has raised concerns about the finances of hysterectomy. ${ }^{6}$ ACOG estimated that the addition of robotic assisted laparoscopic hysterectomy could increase national healthcare spending by as much as $\$ 1.8$ billion. We were unable to isolate the effect of robotic assisted technology on payments, but the increased utilization of laparoscopy did not lead to an increase in overall payments. Two reasons to explain why the change in practice has not led to an increase in expenditures are a $12.5 \%$ decrease in utilization and a shift to outpatient surgery, for which reimbursement is $45 \%$ lower than a similar inpatient procedure.

These analyses also reveal how clinical practice is changing. Between 2010 and 2013, we observed fewer hysterectomies among reproductive-aged women and relatively stable rates among women $>55$. The most common indications for hysterectomy among reproductiveaged women — abnormal uterine bleeding, uterine fibroids, endometriosis, and chronic pelvic pain—decreased markedly. This may be due to the increased use of endometrial ablation and progesterone eluting intrauterine devices to control bothersome abnormal bleeding and chronic pelvic pain. These therapies have enabled many pre-menopausal women to control symptoms without resorting to invasive, extirpative surgery. ${ }^{10}$ The number of hysterectomies for pelvic organ prolapse also decreased $19.4 \%$. Rates of hysterectomy among Medicare beneficiaries diagnosed with prolapse decreased between 1999 and $2009,{ }^{11}$ the decade prior to the period we analyzed and before transvaginal mesh became so commonly used. The decrease we observed may be a continuation of this trend, but it may also be related to Federal Drug Administration Safety Advisories in 2008 and 2011 regarding use of transvaginal mesh for prolapse procedures ${ }^{12}$ Cervical dysplasia, cervical cancer, endometrial hyperplasia, premalignant lesions of uncertain potential, cervical cancer, ovarian cancer, and a family history of cancer affect a much smaller number of women and there was little change in the rates of utilization for those indications. The rate of hysterectomy for endometrial cancer increased $20 \%$. The increase in endometrial cancer, which has an estrogen-dependent disease process, is likely related to rising rates of obesity, which exposes women to higher levels of endogenous estrogen. ${ }^{13}$

There may be an opportunity to reduce total annual payments for hysterectomy with improved adherence to clinical practice guidelines. Vaginal hysterectomy is the least morbid and expensive approach and, when appropriate, is preferred over a laparoscopic or abdominal approach by ACOG. However, during this time, the $11.5 \%$ ). The mean payment for vaginal hysterectomy ranges from $\$ 1,310$ to $\$ 2,859$ less (see Figure 4 ) than that for a 
laparoscopic or laparoscopic assisted vaginal proportion of hysterectomies performed vaginally dropped $31.8 \%$ (from $15.1 \%$ to hysterectomy. While there is gynecologic disease (e.g., large fibroids or adhesive disease due to previous surgery or endometriosis) that might complicate or even contraindicate a vaginal approach, systematic protocols can increase the proportion of vaginal hysterectomies. ${ }^{14,15}$ Programs promoting rational and safe utilization of vaginal hysterectomy could be a responsible strategy for further decreasing expenditures related to hysterectomy.

Geographic variation in hysterectomy utilization rates appears to be diminishing in this analysis, in contrast to earlier studies. Rates of hysterectomy utilization in the United States have traditionally been higher in the South and Midwest than those in the Northeast and West. ${ }^{8,16,17}$ We found that the variation between regions of the country is decreasing significantly, by $48.5 \%$ between 2010 and 2013. This could be due to widespread dissemination of effective treatments that are an alternative to hysterectomy. Methodologic reasons to explain the lower variation could be our use of age adjustment of utilization rates, which was not done in previous analyses, and the fact that women in our analysis had private insurance and were not representative of all payers.

There are other strengths and limitations to consider with this study. When comparing statewide utilization rates, it is important to note that we had to assume women with commercial insurance in the Health Care Cost Institute were similar to those with commercial insurance not in the Health Care Cost Institute and to those without commercial insurance (no insurance or covered by Medicare or Medicaid). The terms of our data use agreement with the Health Care Cost Institute also only provide us access to claims for 2010 through 2013 with three diagnostic codes. We are unable to obtain additional years of data or to receive additional diagnostic codes for claims. Fortunately, the limited number of diagnostic codes is unlikely to affect an analysis of inpatient and outpatient utilization. Second, the payments for hysterectomy describe those of the procedure and the surgical admission but do not account for post-operative adverse events such as medical or surgical complications, readmission, or reoperation after discharge. These events could change our estimate of payments. Third, we decided against including professional payments because they were missing from a large proportion (about one-fourth) of hysterectomies. Fourth, we did not have confidence that we could comment on robotic assisted laparoscopy. The proportion of hysterectomies performed with robotic assistance, identified with ICD9 procedure codes 17.42 and 17.44, was lower than expected in 2010 and decreased markedly over time - a pattern strikingly different from other analyses. ${ }^{2}$ Despite these limitations, an analysis of inpatient and outpatient claims data for 25-26 million commercially insured women of all ages from 50 states provides important data regarding utilization and payments for hysterectomy.

Current hysterectomy practices are characterized by more common use of laparoscopy and a shift toward outpatient surgery. Reproductive-aged women are undergoing fewer hysterectomies - probably due to the availability of less invasive methods of controlling menstrual disorders. Fewer hysterectomies and lower reimbursement rates for outpatient surgery are the most likely reasons for the decrease in total annual payments. 


\section{Supplementary Material}

Refer to Web version on PubMed Central for supplementary material.

\section{Acknowledgments}

The authors acknowledge Kristian Seiler*, for assistance in preparing the United States maps to reflect the state level utilization of hysterectomy and Sarah Block*, for assistance in editing and preparing the manuscript for publication.

*Employed by the University of Michigan; no additional funding or compensation was provided for their contributions to this manuscript.

Source of Funding: National Institute of Child Health and Human Development WRHR Career Development Investigator support for Dr. Carolyn Swenson was provided by the Award K12 HD065257. The NIH did not play a role in the study design; in the collection, analysis, and interpretation of data; in the writing of the report; or in the decision to submit the article for publication.

\section{References}

1. Moore, BJ., Steiner, CA., Davis, PH., Stocks, C., Barrett, ML. Trends in Hysterectomies and Oophorectomies in Hospital Inpatient and Ambulatory Settings, 2005-2013: Statistical Brief \#214. Healthcare Cost and Utilization Project (HCUP) Statistical Briefs. https://www.hcupus.ahrq.gov/ reports/statbriefs/sb214-Hysterectomy-Oophorectomy-Trends.pdf. Accessed 9/19/17

2. Wright JD, Herzog TJ, Tsui J, et al. Nationwide trends in the performance of inpatient hysterectomy in the United States. Obstet Gynecol. 2013; 122(2 Pt 1):233-241. [PubMed: 23969789]

3. Zakaria MA, Levy BS. Outpatient vaginal hysterectomy: optimizing perioperative management for same-day discharge. Obstet Gynecol. 2012; 120(6):1355-1361. [PubMed: 23168760]

4. Maheux-Lacroix S, Lemyre M, Couture V, Bernier G, Laberge PY. Feasibility and safety of outpatient total laparoscopic hysterectomy. JSLS. 2015; 19(1):e2014.00251.

5. Jennings AJ, Spencer RJ, Medlin E, Rice LW, Uppal S. Predictors of 30-day readmission and impact of same-day discharge in laparoscopic hysterectomy. Am J Obstet Gynecol. 2015; 213(3):344.e341347. [PubMed: 25981843]

6. Breeden, JTM. Statement on Robotic Surgery by ACOG President James T. Breeden MD: https:// www.acog.org/About-ACOG/News-Room/News-Releases/2013/Statement-on-Robotic-Surgery. Accessed July 20, 2017

7. The Dartmouth Atlas of Health Care. Indirect Adjustment. http://www.dartmouthatlas.org/ downloads/methods/indirect_adjustment.pdf. Accessed 9/22/17

8. Wu JM, Wechter ME, Geller EJ, Nguyen TV, Visco AG. Hysterectomy rates in the United States, 2003. Obstet Gynecol. 2007; 110(5):1091-1095. [PubMed: 17978124]

9. Desai VB, Guo XM, Fan L, Wright JD, Xu X. Inpatient Laparoscopic Hysterectomy in the United States: Trends and Factors Associated With Approach Selection. J Minim Invasive Gynecol. 2017; 24(1):151-158.e151. [PubMed: 27614151]

10. van Dongen H, van de Merwe AG, de Kroon CD, Jansen FW. The impact of alternative treatment for abnormal uterine bleeding on hysterectomy rates in a tertiary referral center. J Minim Invasive Gynecol. 2009; 16(1):47-51. [PubMed: 18990612]

11. Khan AA, Eilber KS, Clemens JQ, Wu N, Pashos CL, Anger JT. Trends in management of pelvic organ prolapse among female Medicare beneficiaries. Am J Obstet Gynecol. 2015; 212(4): 463.e461-468. [PubMed: 25446663]

12. Sedrakyan A, Chughtai B, Mao J. Regulatory Warnings and Use of Surgical Mesh in Pelvic Organ Prolapse. JAMA Intern Med. 2016; 176(2):275-277. [PubMed: 26713426]

13. Shaw E, Farris M, McNeil J, Friedenreich C. Obesity and Endometrial Cancer. Recent Results Cancer Res. 2016; 208:107-136. [PubMed: 27909905] 
14. Schmitt JJ, Carranza Leon DA, Occhino JA, et al. Determining Optimal Route of Hysterectomy for Benign Indications: Clinical Decision Tree Algorithm. Obstet Gynecol. 2017; 129(1):130-138. [PubMed: 27926638]

15. Schmitt J, Occhino JA, Bakkum-Gamez J, et al. Implementation and evaluation of benign hysterectomy decision tree algorithm. Am J Obstet Gynecol. 2017; 216(3):S574-S575.

16. Lepine LA, Hillis SD, Marchbanks PA, et al. Hysterectomy surveillance-United States, 1980-1993. MMWR CDC Surveill Summ. 1997; 46(4):1-15.

17. Palmer JR, Rao RS, Adams-Campbell LL, Rosenberg L. Correlates of hysterectomy among African-American women. Am J Epidemiol. 1999; 150(12):1309-1315. [PubMed: 10604773] 


\section{Condensation}

Average reimbursement per hysterectomy is increasing, but total payments are decreasing due to decreased utilization and dramatic differences in how hysterectomy is performed. 


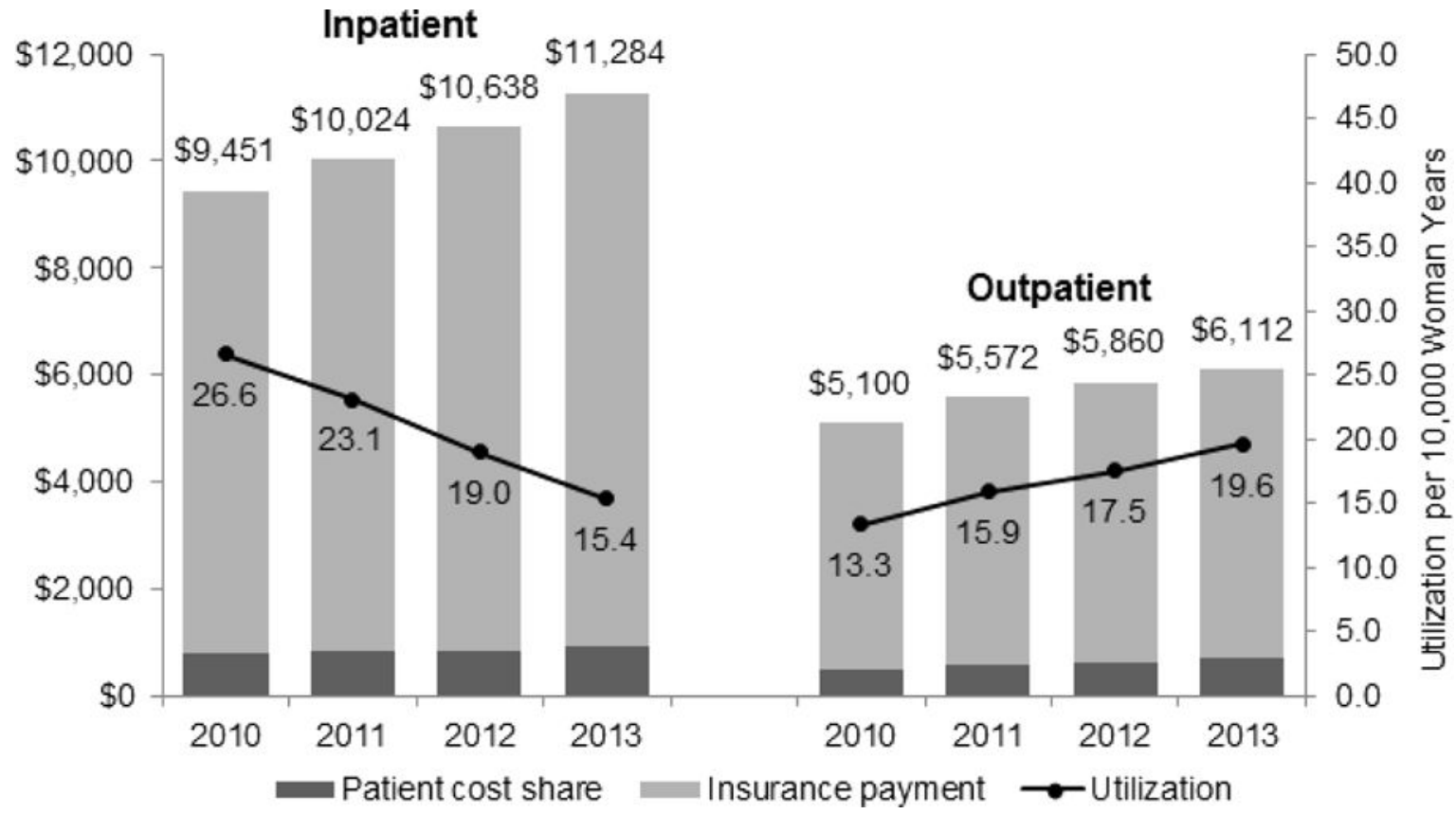

Figure 1. Payment and Utilization for hysterectomy, Health Care Cost Institute, 2010-2013 NOTE: All cost share and payments are adjusted for inflation to 2013 dollars, using the Medical Consumer Price Index. 

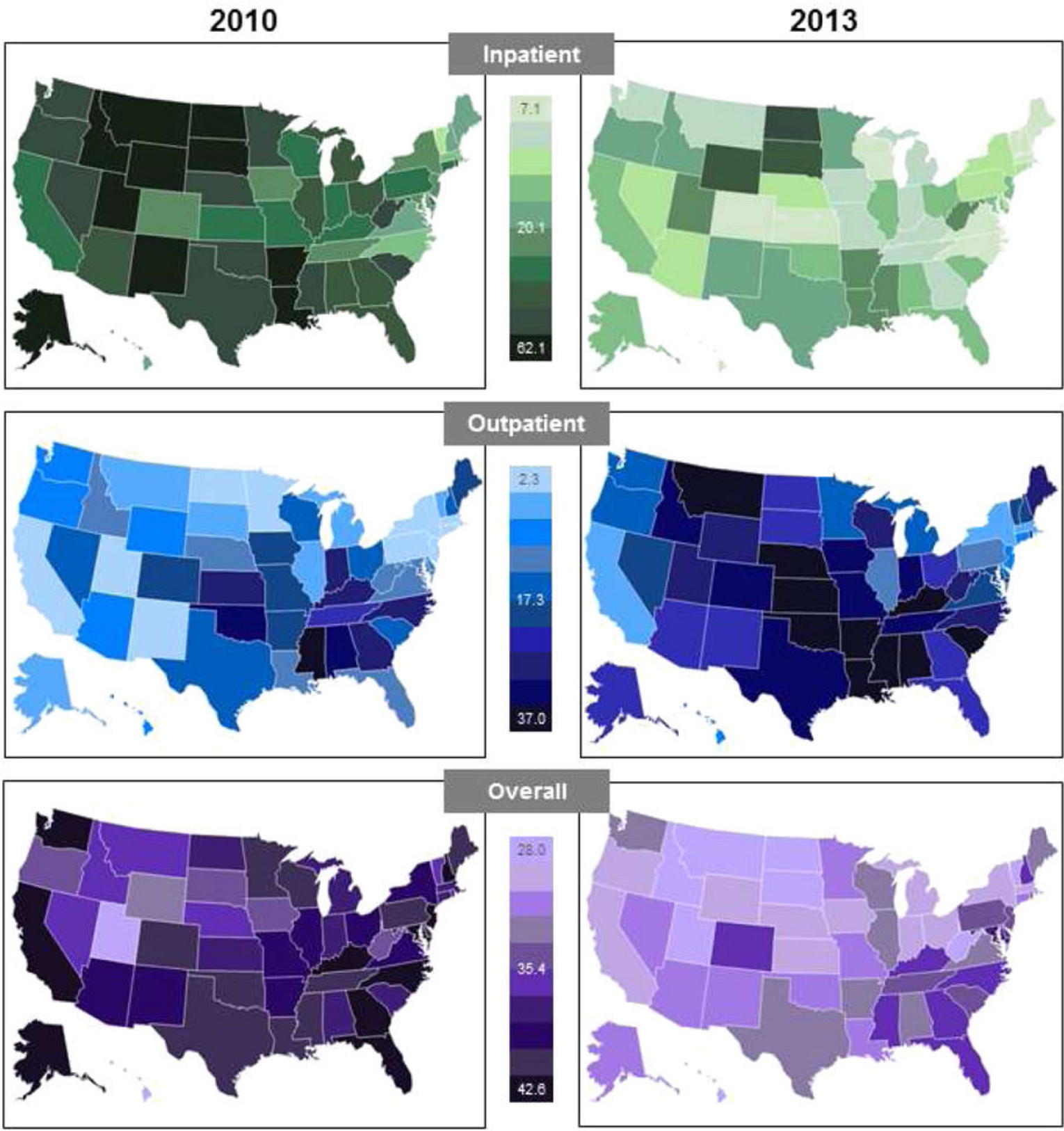

Overall
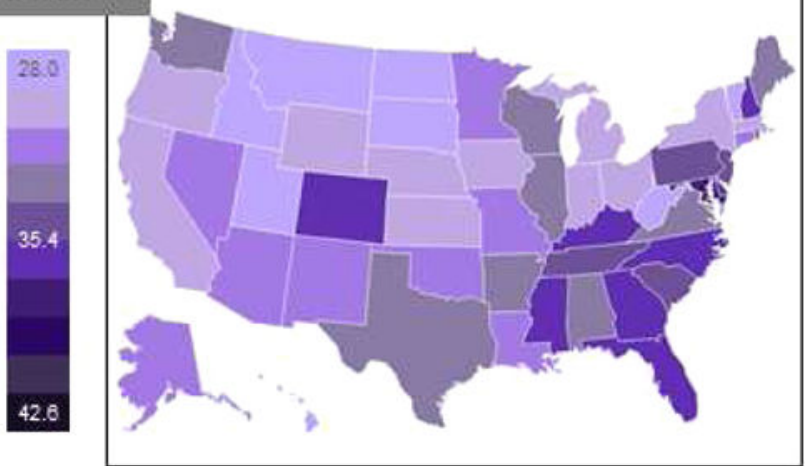

Figure 2. Change in age-adjusted utilization rates of inpatient, outpatient, and all hysterectomies, Health Care Cost Institute, 2010-2013

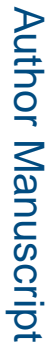
Rates are per 10,000 woman-years. 
$\$ 900,000,000$

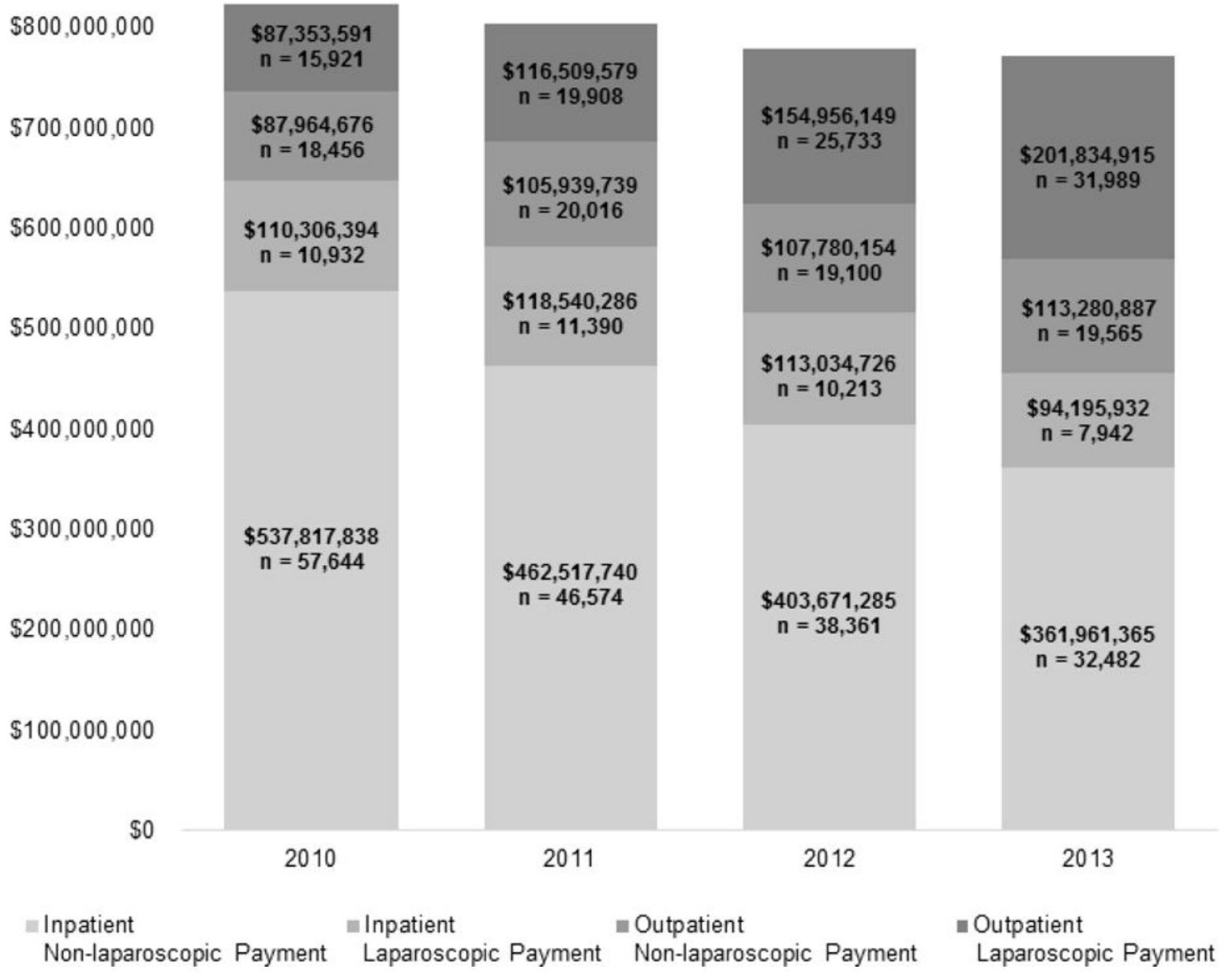

Figure 3. Payments for inpatient and outpatient laparoscopic and non-laparoscopic hysterectomies, Health Care Cost Institute, 2010-2013

LS: Laparoscopic

NOTE 1: Non-laparoscopic hysterectomies are the sum of payments for abdominal, laparoscopically-assisted vaginal, and vaginal hysterectomies. The contribution of each surgical approach is calculated by multiplying the number of hysterectomies by its respective average annual payment.

NOTE 2: All costs are adjusted for inflation to 2013 U.S. dollars, using the Medical Consumer Price Index. 


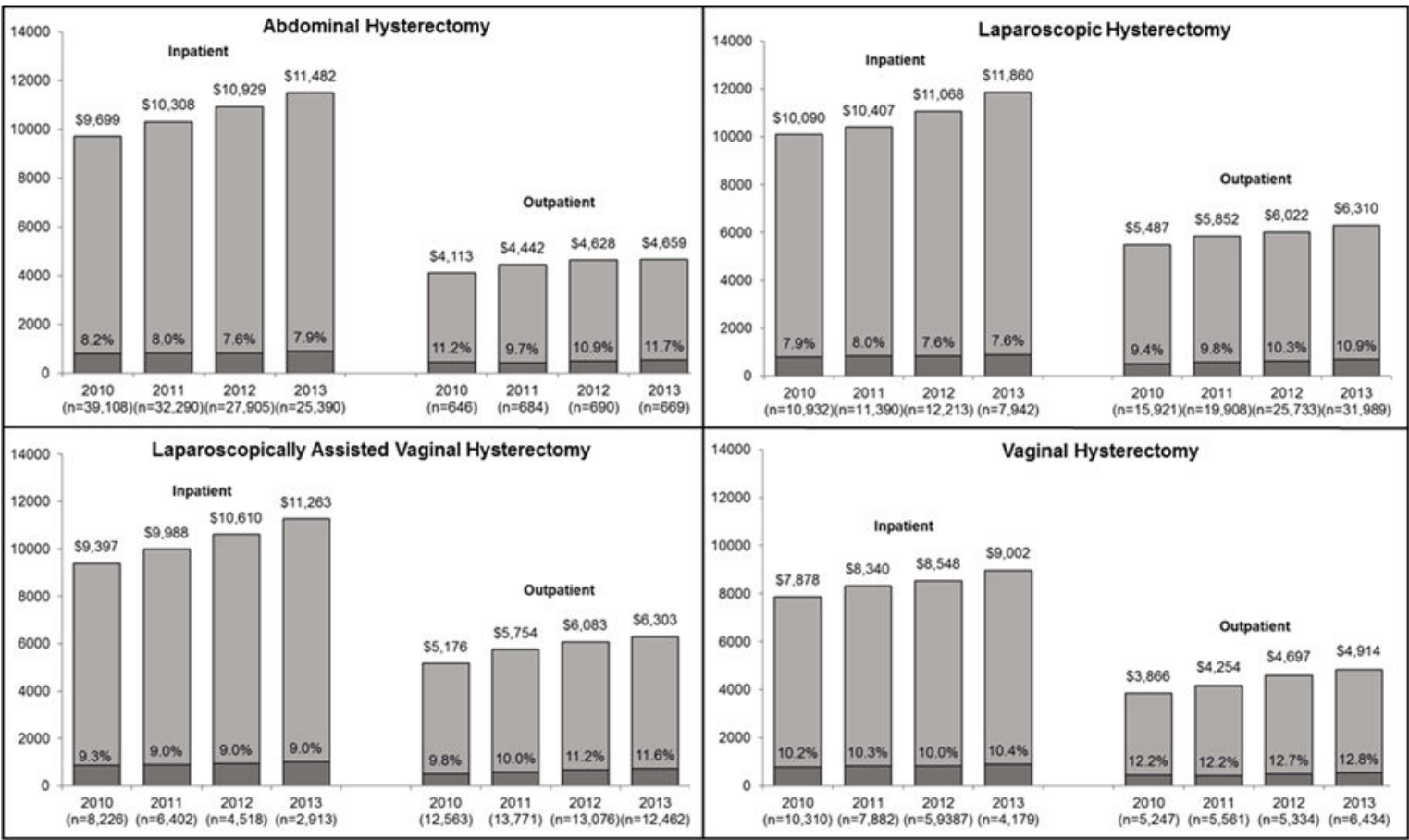

Patient cost share Insurance payment

Figure 4.

Patient and insurance payments for hysterectomy by surgical approach, Health Care Cost Institute, 2010-2013 


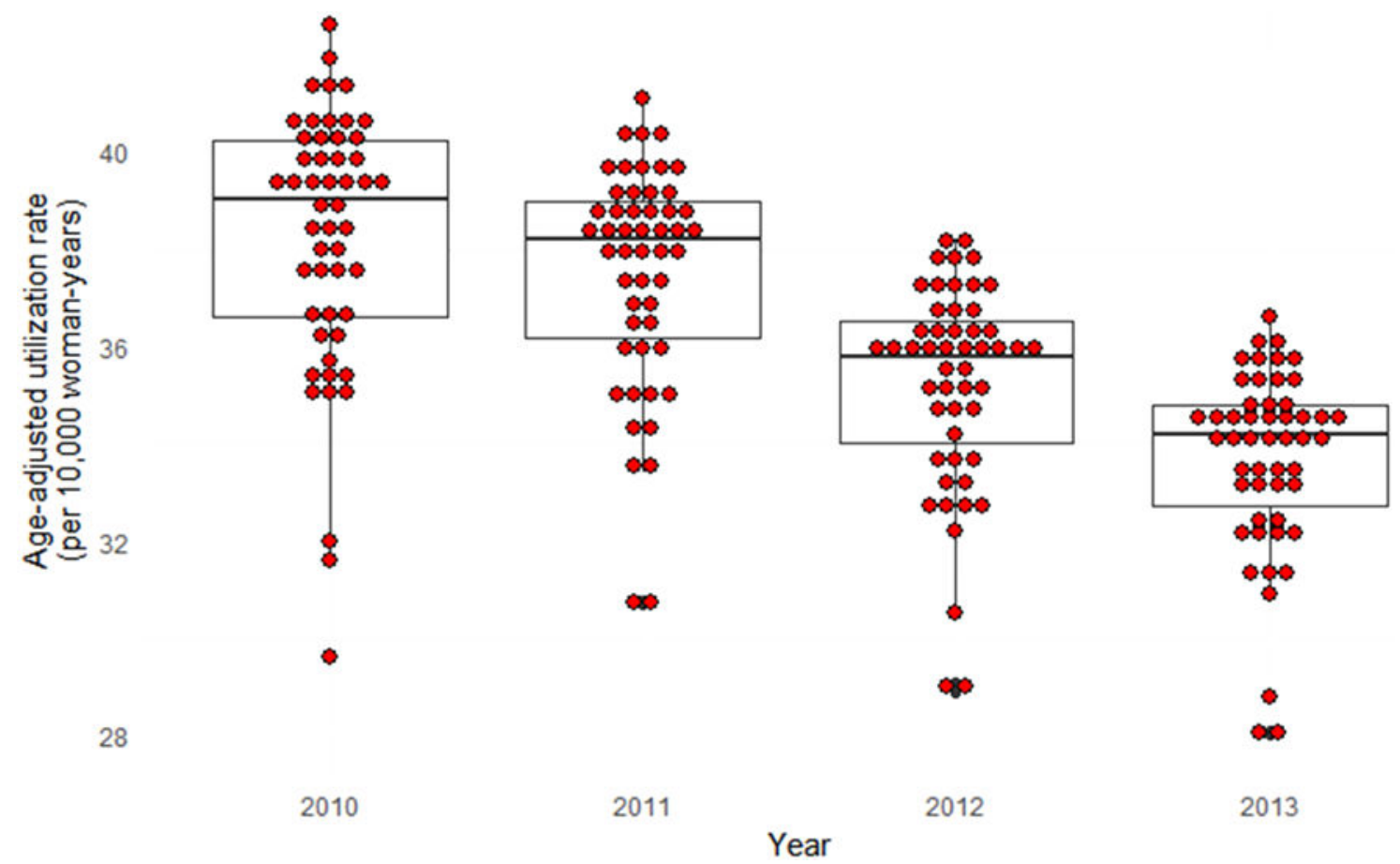

Figure 5. State-level hysterectomy utilization, Health Care Cost Institute, 2010-2013

NOTE: The overall utilization rate for 50 states and the District of Columbia are shown in each year between 2010 and 2013. Boxplot denotes the median and the interquartile range $\left(25^{\text {th }}\right.$ and $75^{\text {th }}$ percentiles $)$. 


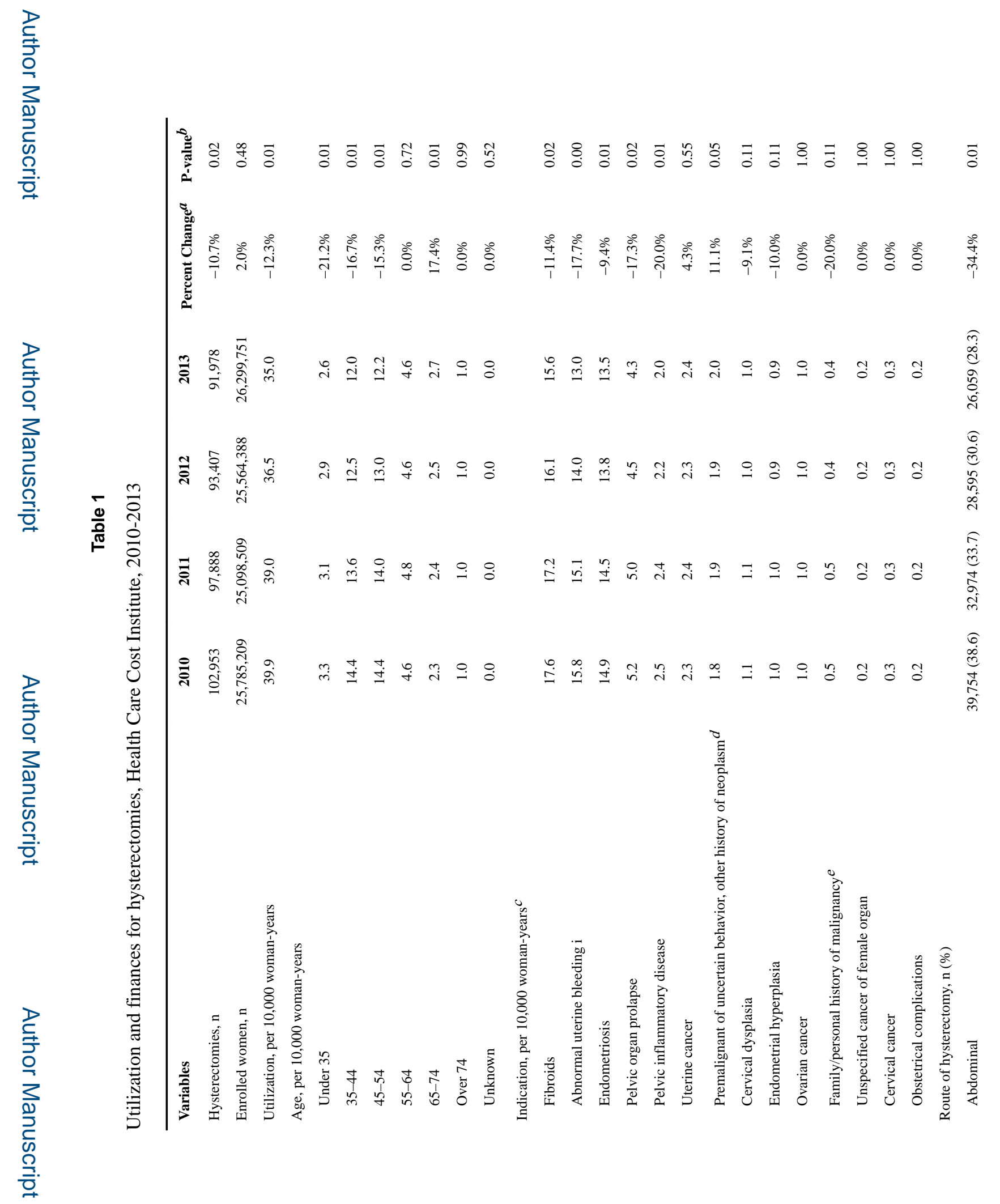

Am J Obstet Gynecol. Author manuscript; available in PMC 2019 April 01. 
MORGAN et al.

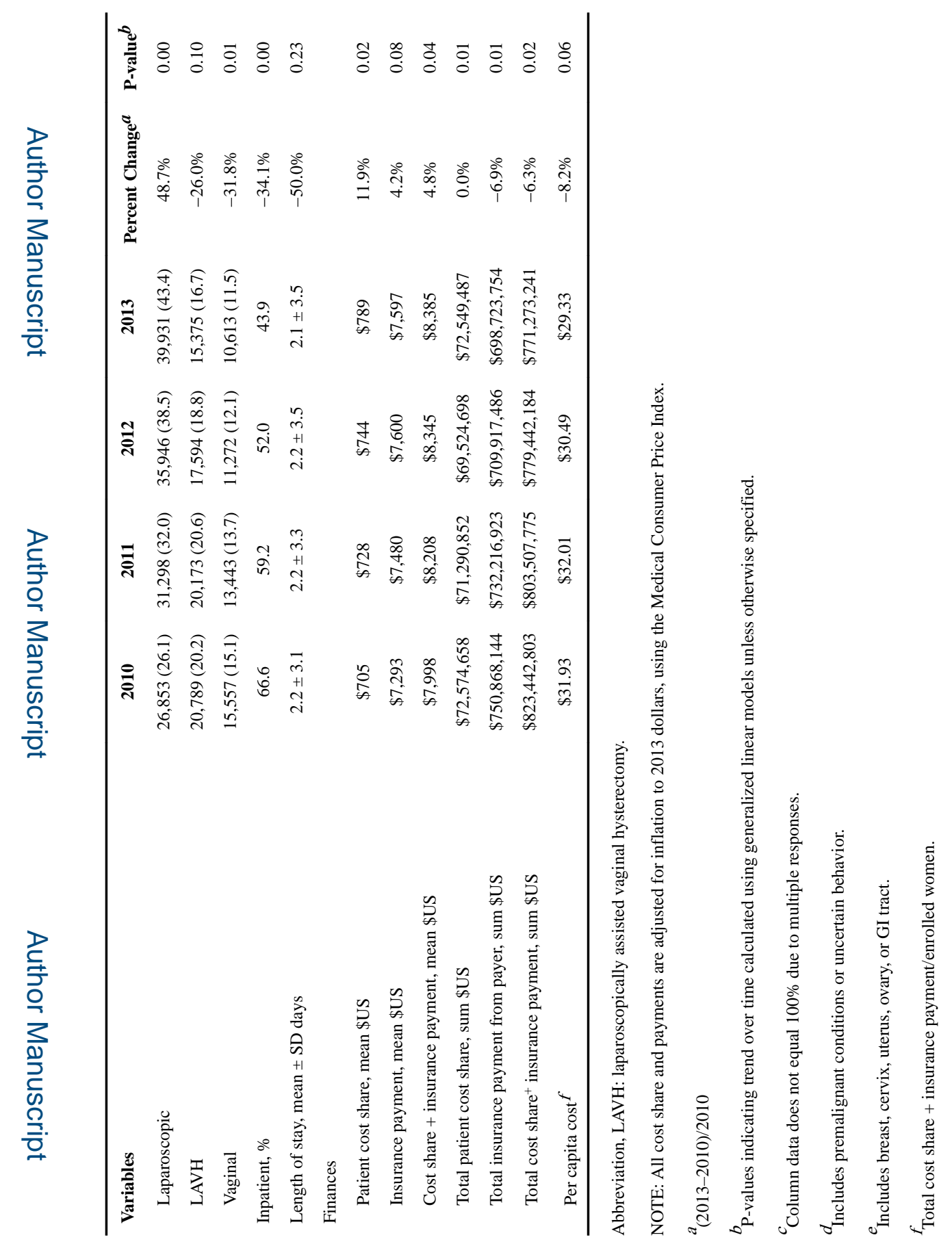

로을

Am J Obstet Gynecol. Author manuscript; available in PMC 2019 April 01. 